\title{
Gas pressurized hot embossing for transcription of micro-features
}

\author{
J.-H. Chang, S.-Y. Yang
}

\begin{abstract}
Hot embossing has proven productive for the parallel replication of precision micro-features onto thermoplastic substrates at low cost. During conventional hot embossing, the substrate and the stamp are brought into contact and are compressed directly by the hot plates of the machine. The accuracy and area of replication are limited due to the inherent non-uniform pressure distribution. Si-wafers are too brittle to be used as embossing tools with the conventional hot embossing operation. This paper describes an innovative pressurizing method for hot embossing using gas as pressure media. The film/stamper/ substrate stack is placed in a closed chamber. After heating, the gas is blown in at high pressure to pressurize the stack. Micro patterns in the stamp can be successfully replicated onto the substrate. Perfectly uniform embossing pressure throughout the whole area can be achieved. Glass or wafers with micro-features on their surfaces can be used as stampers directly.
\end{abstract}

\section{1}

\section{Introduction}

Hot embossing is a productive method for the parallel replication of precision micro-features at low cost. With a single master or stamp, identical structures can be reproduced onto substrates. This technique is widely applied in microstructure fabrication, for example, in optical-sensor and bio-chip applications. So far, many successful replications of the micro-features of stamps have been reported [Gale (1997); Olsson et al. (1998); Becker et al. (2000)]. There are three basic steps in hot embossing. Firstly, a polymer substrate is heated above a certain temperature. Secondly, a mold (stamp or master) is pressed against the substrate, allowing the pattern to be fully transferred onto the substrate (embossing). Finally,

Received: 13 December 2002 / Accepted: 14 January 2003

J.-H. Chang, S.-Y. Yang ( $\square)$

Grace Laboratory for Polymer Processing,

Department of Mechanical Engineering,

National Taiwan University, Taipei,

Taiwan 106, Republic of China

E-mail: syyang@ccms.ntu.edu.tw

The authors would like to thank National Taiwan University for help with lab set-up, Professor Ping-Hei Chen and Mr. Jian Ying Tsai for providing the embossing stamper, and their coworkers at Grace Laboratory for stimulating discussions and experimental assistance. after a suitable amount of contact time between the mold and substrate, the system is cooled down below $T_{\mathrm{g}}$, followed by separating the mold from the substrate (de-embossing). Figure 1 shows schematically the apparatus for a typical hot embossing process.

With conventional hot embossing, the substrate is pressed directly by the hot plates of the press machine. One of the moving hot plates is usually driven by pneumatic cylinder [Juang et al. (2002)], hydraulic cylinder [Chang and Yang (2002)] or linear screw/motor devices [Heckele et al. (1998)]. Some hot embossing facilities use a cylinder pump with adjustable heating blocks [EVGroup (2002)]. Pressing substrates with hot plates has inherent problems. The distribution of the embossing pressure is not uniform. It was found that the embossing pressure was higher in the intermediate zone and lower in the margin zone under thin-film hot embossing [Chang and Yang (2002)]. Similar results were also obtained by Lin et al. (2002). This influences the uniformity of pattern embossing. Furthermore, the shrinkage that occurs during subsequent cooling is affected by the non-uniform pressure distribution, which also greatly limits the replication accuracy and area. Until recently, the maximum wafer size of replication using hot embossing was only 4 inches. The largest working area of a commercial hot embossing machine is limited to $130 \mathrm{~mm}$ [Jenoptik Mikrotechnik (2002)]. Larger areas of uniformity would be highly advantageous to increase throughput, especially for applications such as displays. It has, thus, become more and more urgent and important to solve the problem of non-uniform pressure distribution. In addition, pressing a substrate with hot platens does not allow for the possibility of using stamps made of brittle material. It was reported that silicon molds have the advantage of excellent surface quality and easy mold release [Becker and Heim (2000); Lin et al. (2000), Ong et al. (2002)]. As a result of recent advances in the semiconductor industry, the wafer diameter has increased from 4 to 8 and even 12 inches in just a few years. It would be advantageous to use wafers directly as stamps, but silicon wafers are too brittle to use as stamps in the conventional hot embossing operation. They are easily broken during the embossing stage. This paper reports an innovative method for hot embossing using gas as pressure media. When gas is used as the pressurizing agent, perfectly uniform embossing pressure throughout a large area can be achieved. Glass stamps or silicon wafers with micro-features on their surfaces can be used as stampers directly. 


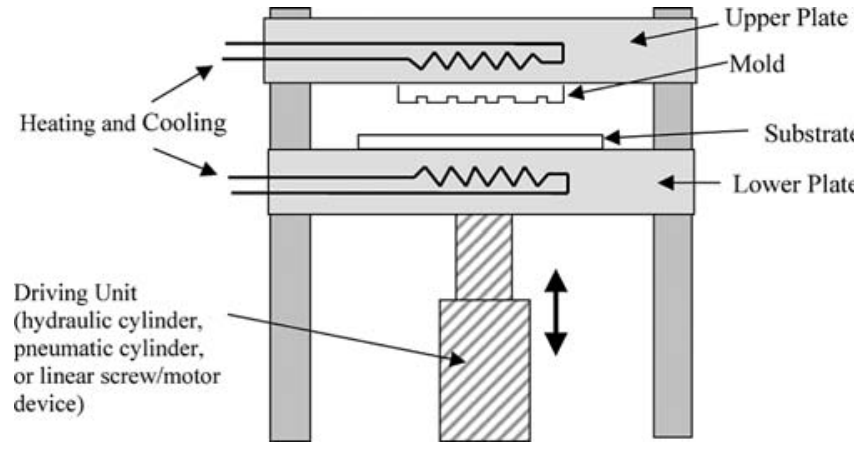

Fig. 1. Schematic drawing of the traditional hot embossing apparatus. (Driving unit: hydraulic cylinder, pneumatic cylinder, linear screw/motor.)

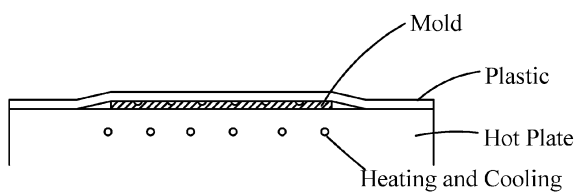

(a) Preparation Material

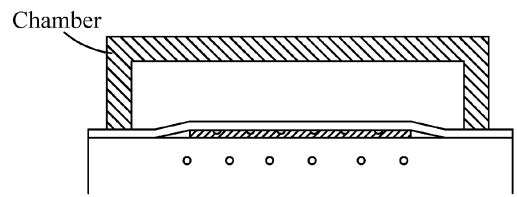

(b) Covering a Chamber

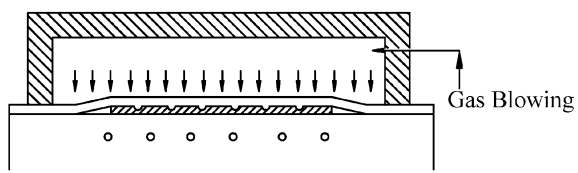

(c) Gas Pressurization (Plate Heating)

\& Gas Packing (Plate Cooling)

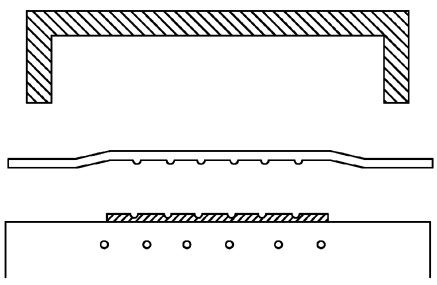

(d) Exhausting \& De-Embossing

Fig. 2. Schematic drawing of the steps of gas pressurized hot embossing process

\section{2}

\section{The gas pressurized hot embossing process}

As illustrated in Fig. 2, the gas pressurized hot embossing process consists of four steps: (1) placing the plastic film onto the stamper to form a film/mold stack, which is then placed above a heating/cooling plate; (2) enclosing the film/mold stacker with a covering chamber, and then heating the film/mold stacker; (3) blowing the gas into the chamber to exert gas pressure uniformly over the film,

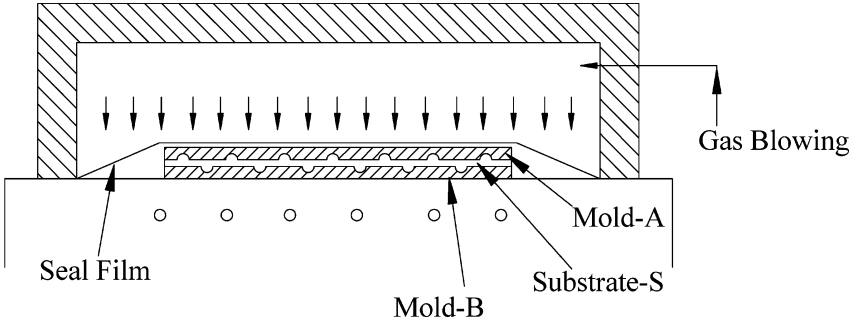

Fig. 3. Schematic diagram showing the double-sided elements or plate embossing. The thicknesses of the mold and substrate are not drawn to scale. (A - Upper mold, B - Lower mold, S - Substrate.)

forcing it into the mold and (4) cooling, and then exhausting the gas and opening the chamber to get the embossed film.

The blown gas can be air or a protective gas, such as nitrogen or argon. In this experiment, nitrogen was used. The gas pressurizing system is composed of a nitrogen tank $\left(120 \mathrm{kgf} / \mathrm{cm}^{2}\right.$ max.), a pressure regulator, valves, and a manual controller. The gas pressure can be regulated with the pressure regulator. Cartridge heaters in the hot plate are used to heat the stamper and polymer substrate, while oil or water is used to cool the system. A vacuum system can be attached to the system if needed.

The embossing master can be a wafer, glass, electroplated Nickel mold, or other stamper with micro-features. The system also allows double-sided embossing onto thermoplastic plates. An additional seal-film as shown in Fig. 3 is used. The function of the seal-film is to permit the external gas pressure to press the mold into the substrate.

During the heating process, low gas pressure is applied to the film to prevent the film from creasing. The thermoplastic film is heated above the glass transition temperature, $T_{\mathrm{g}}$. After the gas pressure is applied, the film is forced to conform to the micro-features in the surface of the stamper. The temperature, the time of embossing, and the applied gas pressure are chosen such that the polymer completely fills the cavities of the stamp during embossing. Once the polymer has conformed to the shape of the stamp, it is cooled to a temperature below $T_{\mathrm{g}}$. Then the gas is vented, the chamber is opened, and the embossed film is retrieved.

\section{1}

\section{Temperature and pressure profiles set-up}

Figure 4 shows the temperature and pressure profiles during the gas pressurized hot embossing process. With respect to the temperature profile, the process starts with the heating stage, remains at the certain fixed temperature stage, and then concludes with the cooling stage. The temperatures depend on the polymeric material used. With respect to the gas pressure profile, two steps of compression are involved. During heating, slight pressure (pre-loading) is applied. After the embossing temperature is reached, high embossing pressure is applied during this constant embossing temperature stage. The embossing pressure can be easily set using the regulator valve. The total cycle time is between 20 and $30 \mathrm{~min}$. 


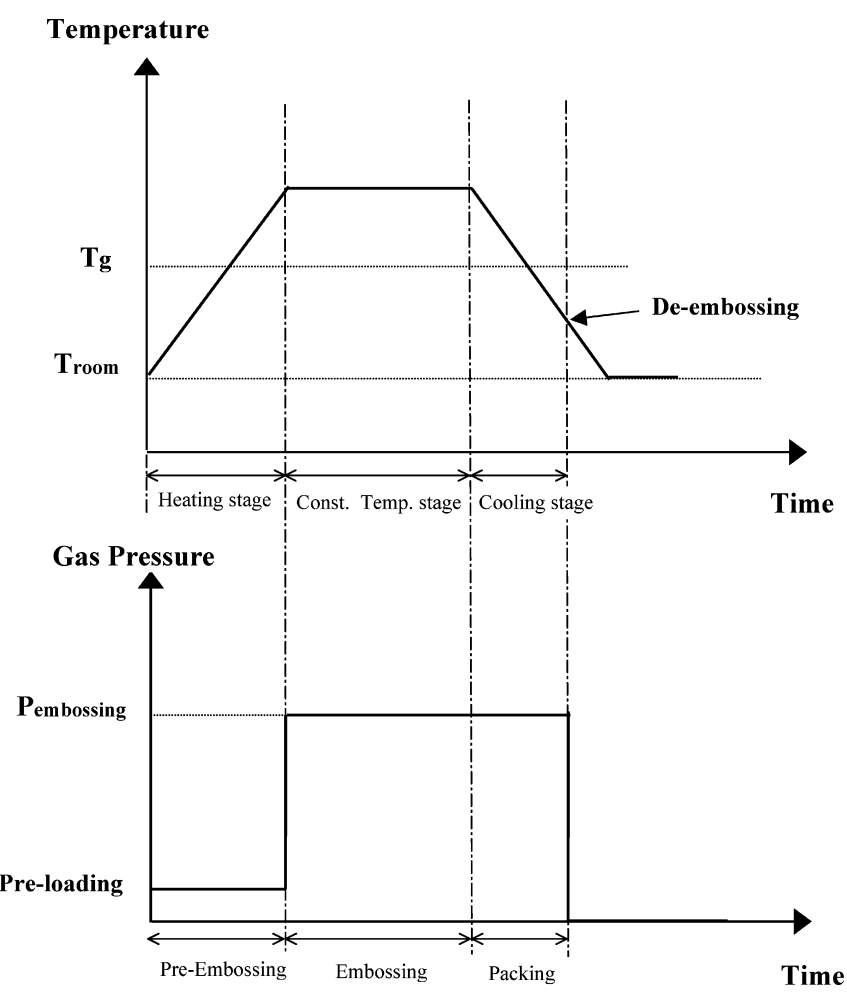

Fig. 4. Temperature and pressure profiles during gas pressurized hot embossing

\section{3}

\section{Test results and discussion}

A 4-inch silicon wafer with micro fluid-channels was used as the mold to characterize the thin-film hot embossing process as shown in Fig. 5. The micro-features of the embossed films were measured using a surface profiler (Alpha-Step 500, TENCOR, USA). Images were also taken using a scanning electronic microscope (Jeol Model 5410, Japan).

\section{1}

\section{Thin-film hot embossing}

Polyvinyl chloride (PVC) film is used in most replication work since it has a lower $T_{\mathrm{g}}\left(=80^{\circ} \mathrm{C}\right)$ temperature. The experiment was carried out at an embossing temperature of $90{ }^{\circ} \mathrm{C}$ with a gas pressure of $40 \mathrm{kgf} / \mathrm{cm}^{2}$. The gas pressure was held constant for $15 \mathrm{~min}$. The thickness of the PVC film was $0.20 \mathrm{~mm}$.

The microstructures were precisely reproduced in the polymeric film as shown in Figs. 6 and 7. The micro-features in the silicon wafer were replicated on the PVC films. No defects due to incomplete filling could be found even with a very low contact gas pressure of $40 \mathrm{kgf} / \mathrm{cm}^{2}$. Gas pressurized hot embossing was proven here to be capable of successfully replicating micro-features in silicon onto thermoplastic films under low gas pressure.

The embossing of thermoplastic films has been reported to be more difficult than that of plates with the conventional platen hot embossing process [Chang and Yang (2002)]. Soft cushion pads are needed between the stamper and upper block to ensure continuous close contact between the stamper and the film throughout the embossing

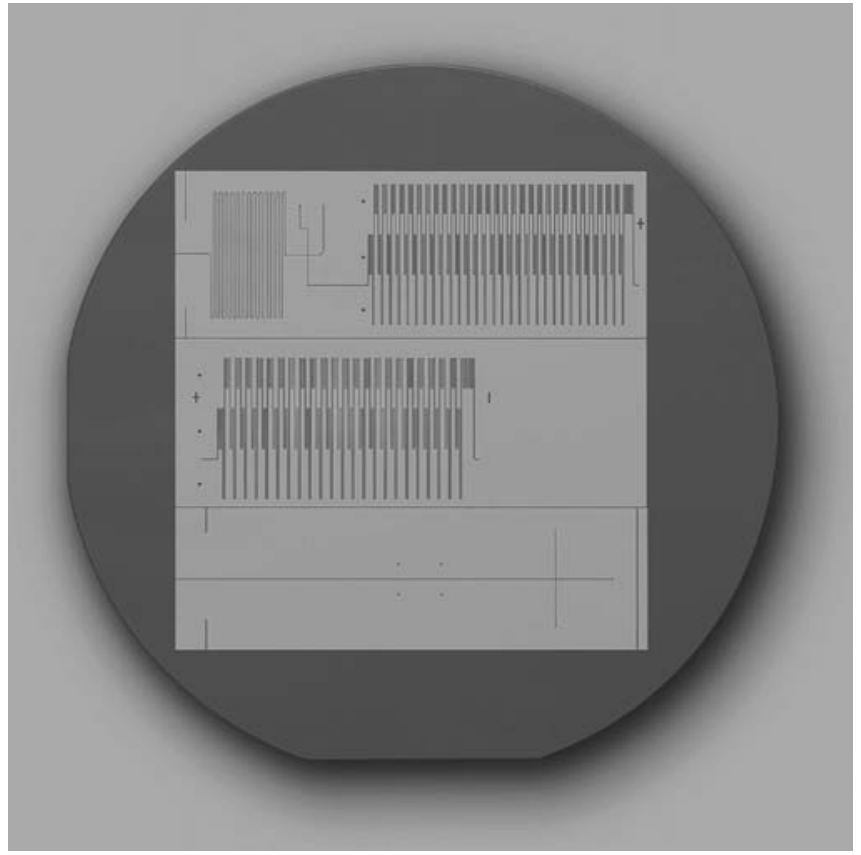

Fig. 5. A whole 4-inch silicon wafer stamper with micro fluidchannels

process. Note that the soft cushion pad is not needed when gas is used as the pressure media in gas pressurized hot embossing.

\section{2}

\section{Substrate hot embossing}

For bio- and MEMS- applications, plastic plate substrates are commonly used in the hot embossing process. Polymethyl methacrylate (PMMA) plates were used in this experiment. The glass transition temperature of the PMMA was around $105{ }^{\circ} \mathrm{C}$. The thickness of the PMMA plates was $2.0 \mathrm{~mm}$. The same 4 -inch silicon wafer was used as the embossing tool. The seal-film used was PET film. The test was carried out at an embossing temperature of $155{ }^{\circ} \mathrm{C}$ with a gas pressure of $40 \mathrm{kgf} / \mathrm{cm}^{2}$. The gas pressure was held constant for $15 \mathrm{~min}$. The results show good transcripted micro-features of the substrates that were hot embossed using gas as the pressurized media. The microfeatures on the silicon could be precisely replicated onto the PMMA plates. Replication of micro-features was successful throughout the whole area. In addition, doublesided elements were also embossed successfully.

\section{4}

\section{Summary and conclusions}

This paper has reported an innovative method for hot embossing using direct gas pressure to press the mold and substrate. A gas pressurized hot embossing facility has been constructed and tested. Successful replication of micro-features on a silicon wafer onto plate substrates and films has been achieved. The embossing pressure was uniform over the whole area. This technique has excellent potential for very large size wafers with microstructures, and it can achieve high replication throughput at, potentially, low cost. The advantages of the gas pressurized hot embossing process can be summarized as follows: 

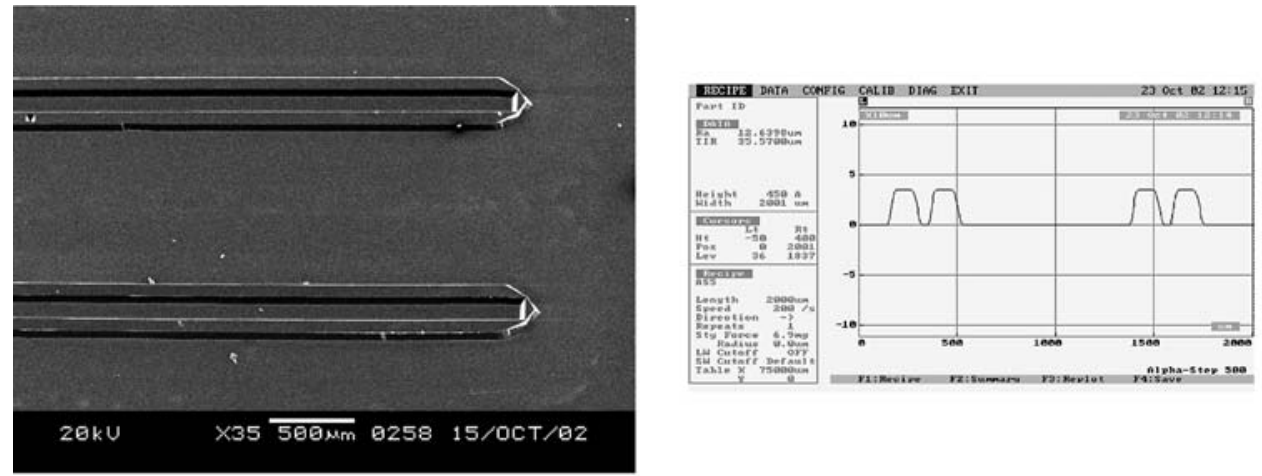

(a) Mold
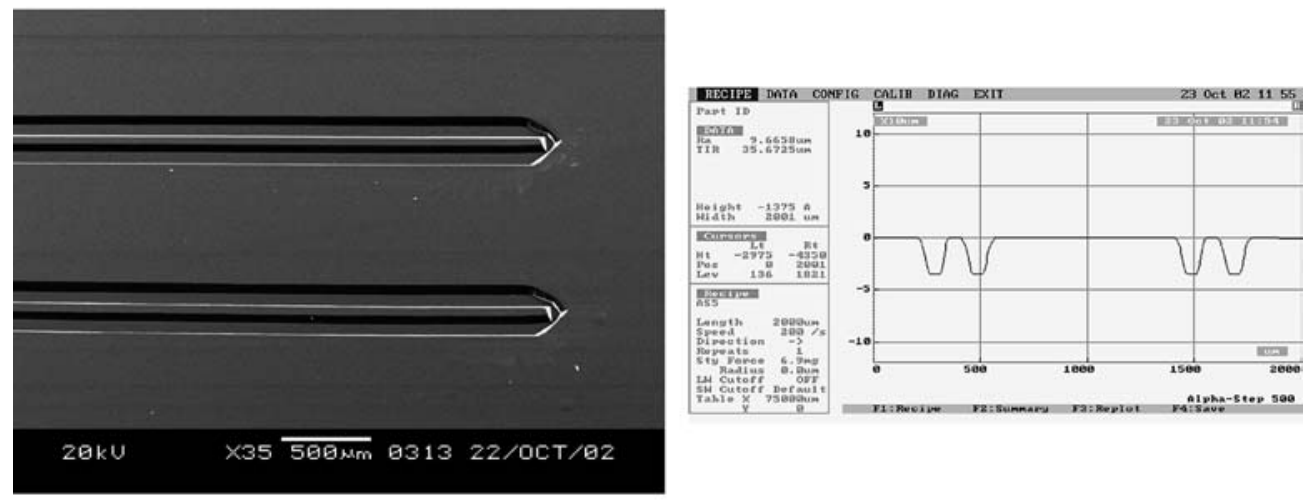

(b) Plastic

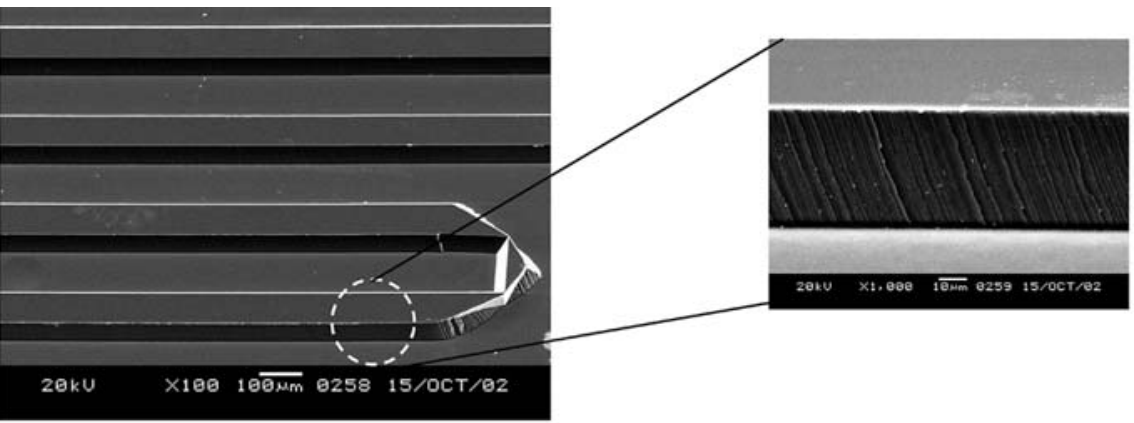

(a) Mold

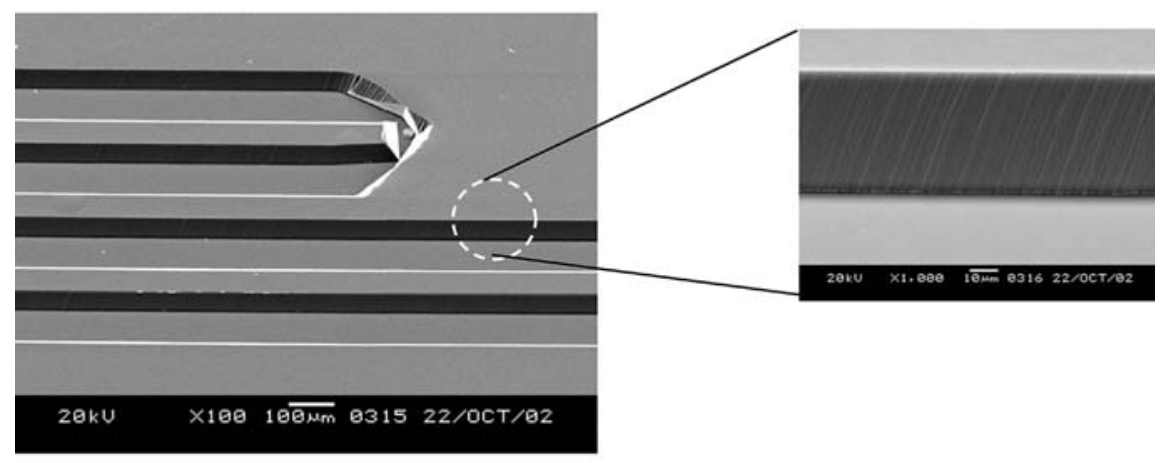

(b) Plastic
Fig. 6. An SEM microphoto and surface profile showing the stamper and replicated micro fluid-channels 
- Compared with the conventional hot embossing process, the new method offers perfectly uniform embossing pressure distribution. This can improve replication accuracy.

- Since the gas is isobaric and isotropic, the embossing area is not limited. Micro-features can be transferred onto large areas (wafers of up to 8 or 12 inches) easily.

- Compared with the conventional hot embossing process, a silicon wafer can be directly used as the embossing tool. The risk of fracturing the wafer tool can be reduced by using gas as the pressure medium.

- The method has also been used to emboss double-sided elements. It is flexible, simple, practical, and reliable.

\section{References}

Becker H; Heim U (1999) Silicon as tool material for polymer hot embossing. Twelfth IEEE International Conference on MEMS '99: 228-231

Becker H; Heim U (2000) Hot embossing as a method for the fabrication of polymer high aspect ratio structures. Sensors and Actuators A 83: 130-135

Chang JH; Yang SY (2002) Hot embossing for transcription of micro-features onto large thermoplastic films. Proceedings of
The Nineteenth National Conference on The Chinese Society of Mechanical Engineers, YunLin, Taiwan

EVGroup (2002) Datasheet of EV520 hot embossing (HE) system of EVGroup

Gale MT (1997) Replication techniques for diffractive optical elements. Microelectronic Eng 34: 321-339

Heckele M; Bacher W; Muller KD (1998) Hot embossing - the molding technique for plastic microstructures. Microsyst Tech 4: 122-124

Jenoptik Mikrotechnik (2002) Datasheet of HEX03 hot embossing system

Juang YJ; Lee LJ; Koelling KW (2002) Hot embossing in microfabrication. part I: experimental. Polymer Eng Sci 42(3): 539550

Lin CR; Chen RH; Hung C (2002) The characterisation and finiteelement analysis of a polymer under hot pressing. Int J Manuf Technol 20: 230-235

Lin L; Shia TK; Chiu CJ (2000) Silicon-processed plastic micropyramids for brightness enhancement applications. J Micromech Microeng 10: 395-400

Olsson A; Larsson O; Holm J; Ohman O; Stemme G (1998) Valveless diffuser micropumps fabricated using thermoplastic replication. Sensors and Actuators A 64: 63-68

Ong NS; Koh YH; Fu YQ (2002) Microlens array produced using hot embossing process. Microelectronic Eng 60: 365-379 